\title{
Cubic and tetragonal liquid crystal phases composed of non-chiral molecules: Chirality and macroscopic properties
}

\author{
Helmut R. Brand ${ }^{1,2, a}$ and Harald Pleiner ${ }^{2, b}$ \\ 1 Department of Physics, University of Bayreuth, 95440 Bayreuth, Germany \\ 2 Max-Planck-Institute for Polymer Research, POBox 3148, 55021 Mainz, Germany
}

Received 17 June 2019 and Received in final form 10 October 2019

Published online: 15 November 2019

(C) The Author(s) 2019. This article is published with open access at Springerlink.com

\begin{abstract}
We discuss the symmetry properties as well as the macroscopic behavior of the cubic liquid crystal phases showing large chiral domains of either hand in some non-chiral compounds reported recently in the group of Tschierske. These phases are tricontinuous. While they have $O$ or $I 432$ symmetry in each chiral domain, the overall symmetry is $\operatorname{Im} \overline{3} m$ as there is no net chirality for compounds composed of non-chiral molecules. It turns out that a rather similar type of phase has also been reported for triblock copolymers. Here we analyze in detail the macroscopic static and dynamic behavior of such phases and we predict, among other results, that they show the analog of static and dissipative Lehmann-type effects in their chiral domains. A description of a cubic liquid crystalline phase of $T_{h}$ symmetry, which has not yet been found experimentally, is also included. Suggestions for experiments are outlined to identify such a phase. In addition, we discuss tetragonal liquid crystalline phases of $D_{4 h}$ and $D_{4}$ (I422) symmetry as they have been reported last year experimentally in connection to the $Q$ phase.
\end{abstract}

\section{Introduction}

Quite recently the group of Tschierske has described and analyzed the observation of chiral domains in several bicontinuous cubic phases composed of achiral molecules $[1$, 2 . The length scale on which these chiral domains are observed is $\sim 500 \mu \mathrm{m}[1,3,4]$, meaning large compared to all molecular length scales and therefore requiring a meso- or macroscopic description of these collective phenomena.

To put the work presented here into a the broader context of more frequent liquid crystalline phases we briefly summarize first the symmetry properties and selected macroscopic aspects of their behavior. For a more detailed exposition we suggest, for example, the book by de Gennes [5]. The most frequently investigated and physically well-understood liquid crystalline phase are uniaxial nematic liquid crystals. These are anisotropic fluid systems with fluidity in 3 dimensions and one preferred direction characterized by a unit vector, that does not distinguish between head and tail, the so-called director, $n_{i}$, which has length unity: $n_{i}^{2}=1$ with $n_{i} \rightarrow-n_{i}$ symmetry. It characterizes quadrupolar orientational order and renders all material properties of uniaxial nematics spatially anisotropic including dielectric properties, electric conductivity, thermal conductivity and viscous properties.

\footnotetext{
a e-mail: brand@uni-bayreuth.de

b e-mail: pleiner@mpip-mainz.mpg.de
}

The deformation energy of this soft material is characterized by three elastic constants for director deformations: splay, twist and bend. Applied voltages of order $1 \mathrm{~V}$ allow deformations of the director field and have been at the basis of applications in liquid crystal displays (LCDs).

When the molecules giving rise to a nematic phase are replaced by chiral molecules and/or when chiral molecules are added to the non-chiral molecules of a nematic phase, a cholesteric liquid crystalline (LC) phase with a helical superstructure with a characteristic repeat distance, the pitch, $P_{0}$, results. The cholesteric phase breaks parity symmetry, since in 3 dimensions it breaks mirror symmetry: left- and right-handed helices are clearly distinguishable from each other. On length scales large compared to the pitch cholesteric LCs are uniaxial [6]. Since many compounds arising in a biological context are chiral, the question of cholesteric phases also naturally comes up in systems as, for example, DNA solutions. We mention in passing that for both, nematic and cholesteric phases, there is a biaxial analogue with two rather than one preferred direction for the orientational order: biaxial nematics [5] and biaxial cholesterics [7].

Major other classes of liquid crystalline phases include layered phases (smectic phases) with varying degree of order within or from layer to layer, but always with positional order (denisty wave) in at least one direction and columnar phases with density waves in at least two dimensions. The simplest phase in this context is the smectic $A$ 
phase with fluidity with isotropic fluidity in 2 dimensions. Depending on the field (for example micellar or biological systems) this type of phase is also called $L_{\alpha}$ or neat soap.

Approaching the liquid crystalline systems of interest here we refer to cubic liquid crystalline phases of various types including $D$ phases [8], bicontinuous cubic phases in a lyotropic and biological context $[9,10]$ and cholesteric blue phases [11-13]. The characteristic length scale of the unit cell varies from about $10 \mathrm{~nm}$ ( $D$ phases) to about $300 \mathrm{~nm}$ (cholesteric blue phases) reflecting the fact that the number of molecules in a unit cell is varying by about five orders of magnitude.

The observations of Tschierske's group are made for bicontinuous cubic liquid crystals of three networks and $\operatorname{Im} \overline{3} m\left(O_{h}\right)$ symmetry. Within each chiral domain one encounters $I 432$ or $O$ symmetry. On the other hand, bicontinuous cubic liquid crystals of two networks and $I a \overline{3} d\left(O_{h}\right)$ symmetry do not show chiral domains and are achiral $[3,4]$.

In retrospect ${ }^{1}$ it appears that also early work of about two decades ago [14] shows chiral domains of three networks in a bicontinuous cubic liquid crystal phase.

In the field of $A B C$ triblock copolymers [15-17] cubic bicontinuous triblock copolymers have also been investigated ("double gyroid structures") and a tricontinuous double gyroid structure of I432 symmetry [17] has been identified. We note ${ }^{2}$, however, that it appears not feasible to observe macroscopic chiral domains in the presently available triblock copolymer systems.

To describe cubic orientational order a fourth-rank traceless symmetric tensor, $Q_{i j k l}[18-20]$ (also called unitary nonor [19]) has been introduced. For a detailed discussion of several of the physical properties and various phase transitions involving achiral cubic phases we refer to [18]. The implications of such an order parameter for transient cubic orientational order above the glass transition have been addressed in [20]. The group theoretical aspects and their physical implications of phases with $O$ and $O_{h}$ symmetry have been elucidated in refs. [21] and [22].

In contrast to other members of the cubic group such as the tetrahedral phases with $T_{d}$ or $T$ symmetry [19,22-27], the macroscopic structure of phases with $O_{h}$ and $O$ symmetry do not break parity (inversion symmetry) $[19,21]$ via the presence of octupolar order. However, the $O$ phase is chiral, possesses a pseudoscalar $q_{0}$ but does not show piezoelectricity. As has been noted in ref. [19], the fourth-rank symmetric and traceless order parameter $Q_{i j k l}$ is suitable for the description of the structure of the achiral $O_{h}$ phase as well as for the chiral $O$ phase.

Motivated by the observation of macroscopic chiral domains in cubic bicontinuous phases of $I 432(O)$ symmetry, we present in the bulk of this paper the macroscopic dynamics of such a chiral phase. We find that it allows for the existence of static and dynamic Lehmann effects in each chiral domain separately while the overall symmetry

\footnotetext{
${ }^{1}$ It is a pleasure to thank Carsten Tschierske for correspondence.

${ }^{2}$ It is a pleasure to thank Volker Abetz for correspondence.
}

of the whole system is of $I m \overline{3} m$ symmetry and thus cubic, but achiral.

The existence of a pseudoscalar quantity $q_{0}$ in the $O$ phase of chiral cubic liquid crystals can be traced back to the fact that one has three networks joined together, microscopically. This gives rise to a chiral arrangement locally even for non-chiral molecules [1-4], which in turn can lead to chiral domains of macroscopic size. This process has been called "propagation of homochiral twist across the entire networks through helix matching at network junctions" in ref. [1]. In the case of three networks there are two possibilities of joining them leading to either hand microscopically, thus defining $q_{0}$ and $-q_{0}$ as pseudoscalar quantities. A similar reasoning concerning the origin of chirality applies to triblockcopolymers [15-17] (see footnote $^{2}$ ).

This situation should be contrasted to the type of structural chirality encountered, for example, for bentcore molecules in the smectic $\mathrm{C}_{B 2}$ (B2) phase of bentcore liquid crystals [28-30]. In this case the way bent-core molecules are arranged macroscopically, i.e. the orientation of the polar direction and the tilt of the director with respect to the smectic layers, leads to chirality of either hand. This has been called "ambidextrous chirality" [30].

It is a hallmark of structurally chiral phases that both types of handedness are equal in the sense that there is no energetic preference for one or the other.

We also investigate the order parameter and the hydrodynamics of the $T_{h}$ phase, the fifth member of the group of cubic phases (compare, for example, ref. [21]). The $T_{h}$ phase is not breaking parity, and does not show any fourfold axes of rotation. We will discuss how the order parameter of $T_{h}$ is different from $O_{h}$ and suggest experiments how to distinguish $O_{h}$ and $T_{h}$ practically. We also mention some aspects regarding the chiral $T$ phase in relation to the chiral $O$ phase.

Among the tetragonal phases of interest are in particular $D_{4 h}$ and $D_{4}$, since phases of these symmetries have been identified recently [31] in connection with the exotic $Q$ phase. Originally found several decades ago [32], for chiral materials [32,33], also a $Q$ phase composed of achiral molecules exists [31]. For chiral compounds its symmetry has been identified as $I 422\left(D_{4}\right)$ in ref. [34]. In the case of the $Q$ phase of achiral components, it is shown experimentally that there are domains of both handedness, $q_{0}$ and $-q_{0}$. Here, the chirality of $D_{4}$ is brought out by the microscopic model discussed in ref. [31].

The paper is organized as follows. In sect. 2 we discuss in detail the symmetries and the hydrodynamic variables of the $O_{h}$ and $O$ phases as well as their differences, followed by the symmetry and the hydrodynamic variables of $T_{h}$ and $T$ in sect. 3 and of $D_{4 h}$ and of $D_{4}$ in sect. 4 . In sect. 5 we derive hydrodynamic equations focusing in particular on the chiral $O$ phase and its physical differences from the achiral $O_{h}$ phase. In sect. 6 we discuss the hydrodynamics of the $T_{h}$ (and partly the $T$ ) phase and in sect. 7 the hydrodynamics of the $D_{4 h}$ and $D_{4}$ phases. We conclude in sect. 8 by a summary of the main results and their connection to experiments. 


\section{Symmetries and symmetry variables of the $\mathrm{O}_{\mathrm{h}}$ and the $\mathrm{O}$ phase}

The $O_{h}$ phase in cubic liquid crystals can be viewed as a cubic biaxial nematic such that the (mutually orthogonal) directors $\boldsymbol{l}, \boldsymbol{m}, \boldsymbol{n}$ are along the edges of the cube. As directors they are normalized, e.g. $\boldsymbol{n}^{2}=1$, and show the equivalence of, e.g., $\boldsymbol{n} \rightarrow-\boldsymbol{n}$, etc. The three directors are all equivalent. It should be noted that those directors are not preferred directions in the nematic sense, since none of them can be detected by optical inspection. For the cubic order parameter our notation follows closely refs. [18, 19]. Specifically, we have

$$
\begin{aligned}
Q_{i j k l}(\mathbf{r})= & Q_{0}\left[l_{i} l_{j} l_{k} l_{l}+m_{i} m_{j} m_{k} m_{l}+n_{i} n_{j} n_{k} n_{l}\right. \\
& \left.-\frac{1}{5}\left(\delta_{i j} \delta_{k l}+\delta_{i k} \delta_{j l}+\delta_{i l} \delta_{j k}\right)\right]
\end{aligned}
$$

with the cubic triad $l_{i}, m_{i} n_{i}$ and the normalization $Q_{0}^{2}=\frac{5}{6} Q_{i j k l} Q_{i j k l}$. The four-fold symmetry axes are given by $\boldsymbol{l}, \boldsymbol{m}, \boldsymbol{n}$ and $\boldsymbol{l} / \boldsymbol{m}, \boldsymbol{m} / \boldsymbol{n}$, and $\boldsymbol{n} / \boldsymbol{l}$ are mirror planes. The three-fold symmetry axes are the space diagonals of the cube. It is not possible to construct out of $Q_{i j k l}$ any tensor of rank 3 or lower, except for the isotropic Kronecker symbol $\delta_{i j}$, thus excluding uniaxial nematic and polar order.

For the chiral $O$ phase the mirror planes of the $O_{h}$ phase are removed and only the symmetry axes remain. In the $O$ phase the chirality is manifest by the existence of a pseudoscalar quantity $q_{0}$ that is of microscopic origin, either due to chiral molecules or due to local chiral arrangements of achiral molecules as discussed in the Introduction. We will adopt the description of ref. [6], where the hydrodynamics of the chiral state is that of the achiral one amended by all possible contributions allowed by $q_{0}$. This is similar to the case of a cholesteric liquid crystal arising from a nematic one, when molecular chirality is added.

In the $O$ and the $O_{h}$ phase the three independent rotations of the rigid structure are the hydrodynamic degrees of freedom (Goldstone modes), like in any other phase with spontaneously fully broken rotational symmetry, i.e. biaxial nematic phases $[35,36]$ and the $T_{d}$ phase $[26,37]$. In equilibrium $Q_{i j k l}$ is constant. Changes of that structure, $\delta Q_{i j k l}$, generally contain internal deformations, in addition to the rigid rotations of the cubic structure. The former are non-hydrodynamic and will not be considered here. The latter are the symmetry variables given by

$$
\delta \Theta_{i} \equiv \frac{5}{2 Q_{0}^{2}} Q_{p j k l} \epsilon_{i p q} \delta Q_{q j k l}
$$

The definition (2) applies to any 1st-order differential $\delta$ and can be inverted, $\delta Q_{i j k l}=2 \epsilon_{i q p} Q_{p j k l} \delta \Theta_{q}$.

For infinitesimal rotations, where in the definition eq. (2) $Q_{p j k l}$ can be replaced by its equilibrium value, the three angles $\Theta_{i}$, describing infinitesimal rotations about a direction $d_{i}$, form an axial vector. In the general case of finite rotations consequent rotations about different direc- tions do not commute, and the conditions

$$
\begin{aligned}
\left(\delta_{1} \delta_{2}-\delta_{2} \delta_{1}\right) \Theta_{i} & =\frac{5}{Q_{0}^{2}} \epsilon_{i p q}\left(\delta_{1} Q_{p j k l}\right)\left(\delta_{2} Q_{q j k l}\right) \\
& =2 \epsilon_{i p q}\left(\delta_{1} \Theta_{p}\right)\left(\delta_{2} \Theta_{q}\right)
\end{aligned}
$$

apply $[35,38]$ indicating $\Theta_{i}$ not being a vector.

There is an alternative way of describing the hydrodynamics of the $O_{h}$ and $O$ phase [36] by using the rotations of the individual directors. One possible representation is

$$
\delta \Theta_{i}=\epsilon_{i j k}\left(l_{j} \delta l_{k}+n_{j} \delta n_{k}+m_{j} \delta m_{k}\right),
$$

where the equivalence of the rotations of $\boldsymbol{l}, \boldsymbol{m}$, and $\boldsymbol{n}$ is apparent. The expression (4) can only be used together with the conditions that guarantee orthogonality among the directors. In order to discuss differences between an isotropic state and the $O_{h}$ phase, which is optically isotropic, the use of $\delta \Theta_{i}$, however, is more suitable.

\section{Symmetry and symmetry variables of the $T_{h}$ and the $T$ phase}

While the $O_{h}$-phase and more recently also the $O$-phase have become of experimental relevance as symmetries in the field of liquid crystals, the third phase of the cubic family, which does not break parity symmetry, namely the $T_{h}$ phase, has not been reported as yet experimentally. One of the goals of the present paper is therefore to find out how one could distinguish a liquid crystalline phase with $O_{h}$ symmetry from a phase with $T_{h}$ symmetry experimentally. To do this we first analyze how the order parameter for the $T_{h}$ phase must be different form the order parameter $Q_{i j k l}$ used to characterize the $O$ and the $O_{h}$ phases discussed above. The difference in symmetry is related to the fact that $T_{h}$ has no 4 -fold axes of rotations. We are therefore looking for an order parameter which accounts for this lower symmetry when compared to $O_{h}$ and $O$, but nevertheless has cubic symmetry and does not break parity such as $T_{d}$ and $T$. Inspecting the structure of $Q_{i j k l}$ one realizes that one can construct easily the fourth-rank tensor $Q_{i j k l}^{T}$

$$
\begin{aligned}
Q_{i j k l}^{T}= & Q_{0}^{T}\left[l_{i} l_{j}\left(m_{k} m_{l}-n_{k} n_{l}\right)+m_{i} m_{j}\left(n_{k} n_{l}-l_{k} l_{l}\right)\right. \\
& \left.+n_{i} n_{j}\left(l_{k} l_{l}-m_{k} m_{l}\right)\right] .
\end{aligned}
$$

Note that $Q_{k l i j}^{T}$ is antisymmetric, when $\{k l\}$ and $\{i j\}$ are interchanged.

By construction, $Q_{i j k l}^{T}$ still has $\boldsymbol{l}, \boldsymbol{m}, \boldsymbol{n}$ as symmetry axes, which are now twofold axes of rotation, rather than fourfold ones, as they are for $O_{h}$. Thus we can reduce the symmetry from $O_{h}$ to $T_{h}$ by adding $Q_{i j k l}^{T}$ as order parameter in the $T_{h}$ phase. Therefore $Q_{i j k l}^{T}$ can serve as the order parameter for describing the $O_{h} \rightarrow T_{h}$ phase transition.

The order parameter $Q_{i j k l}^{T}$ does not introduce any new variables, and the three rotations of the cube of eq. (2) can here be written as $\delta \Theta_{i} \sim Q_{p j k l}^{T} \epsilon_{i p q} \delta Q_{q j k l}^{T}$. Although 
the variables in the $O_{h}$ and the $T_{h}$ phase are the same, there are differences in the form of the material tensors, regarding, e.g., Frank elasticity, and electro- and magnetostriction (for liquid crystalline elastomers and gels). The terms electrostriction and magnetostriction refer to coupling terms in the energy, which are linear in the strain and quadratic in the electric or magnetic field. They are of the form

$$
\varepsilon_{E S}=\chi_{i j k l}^{E} \varepsilon_{i j} E_{k} E_{l},
$$

where $\varepsilon_{i j}$ denotes the tensor of the strain field and $E_{i}$ the electric field. Thus electrostriction and magnetostriction are associated with a fourth-rank material tensor, which is symmetric in the first pair of indices (symmetry of the strain tensor) as well as in he second pair of indices separately. Applying an electric field thus leads to an elongation/shortening of the sample, which is quadratic in the electric field.

If a $T_{h}$ phase gets chiralized, e.g., by the presence of a pseudoscalar of microscopic origin, the mirror planes are removed and the phase has $T$ symmetry. The same phase is obtained, when a tetrahedral $T_{d}$ phase gets chiralized. The hydrodynamics of such a chiral $T$ phase has been described earlier [27,37] and will not be repeated here. Only a few remarks on the differences between the $T_{h}$ and the $T$ phase will be given.

\section{Symmetry and symmetry variables of the $D_{4 h}$ and $D_{4}$ phase}

Both, the $D_{4 h}$ and the $D_{4}$ phase are of tetragonal symmetry. Therefore both can be characterized by a preferred direction, which we represent by a director $n_{i}$. It is also associated with uniaxial nematics and can be extracted from the quadrupolar order parameter

$$
Q_{i j}=S\left(n_{i} n_{j}-\frac{1}{3} \delta_{i j}\right) .
$$

This observation allows us to use as invariants in both phases, $D_{4 h}$ and $D_{4}$, the director $n_{i}$ and the transverse Kronecker delta, $\delta_{i j}^{\perp}=\delta_{i j}-n_{i} n_{j}$. This is all what is needed to describe the rank-2 material tensors.

For the rank-4 material tensors the four-fold symmetry in the plane perpendicular to $n_{i}$ requires a suitable rank- 4 order parameter. In principle, one could use $Q_{i j k l}$ familiar from the $O_{h}$, and $O$ phases. A more specific and convenient choice is the projection of the cubic order parameter onto the plane $\perp n_{i}$

$$
\begin{aligned}
Q_{i j k l}^{t r}= & Q_{0}\left[l_{i} l_{j} l_{k} l_{l}+m_{i} m_{j} m_{k} m_{l}\right. \\
& \left.-1 / 4\left(\delta_{i j}^{\perp} \delta_{k l}^{\perp}+\delta_{i k}^{\perp} \delta_{j l}^{\perp}+\delta_{i l}^{\perp} \delta_{j k}^{\perp}\right)\right],
\end{aligned}
$$

where $l_{i}$ and $m_{i}$ are directors describing a square in the plane $\perp n_{i}$ with the normalization $Q_{0}^{2}=2 Q_{i j k l}^{t r} Q_{i j k l}^{t r}$. With the help of the transverse directors, the transverse Kronecker delta can also be written as $\delta_{i j}^{\perp}=m_{i} m_{j}+l_{i} l_{j}$, reflecting the tetragonal symmetry, i.e. invariance under a transformation $\boldsymbol{m} \rightarrow \boldsymbol{l} \wedge \boldsymbol{l} \rightarrow-\boldsymbol{m}$.
While $D_{4 h}$, also called $\frac{4}{m} \mathrm{~mm}$ or $\frac{4}{m} \frac{2}{m} \frac{2}{m}$, has horizontal mirror planes, they are absent in the chiral $D_{4}$ phase (also called 422), due to the presence of a pseudoscalar $q_{0}$. Particularly, it is well known [22] that the $D_{4}$ phase shows piezoelectricity requiring a parity-breaking third-rank tensor, which can be constructed using $q_{0}$ and $n_{i} n_{p} \epsilon_{p j l} \delta_{k l}^{\perp}$.

The piezoelectric effect is, in general, a static coupling in the energy between the strain field and an electric field, which takes the form

$$
\varepsilon_{p}=\zeta_{i j k}^{p} \varepsilon_{i j} E_{k}
$$

It requires a ground state that breaks parity (inversion) symmetry such as, for example, quartz [22]; $\zeta_{i j k}^{p}$ is symmetric in $i$ and $j$ due to the symmetry of the strain field. In contrast to electrostriction, piezoelectricity is an effect in an external electric field. Conversely, applying a strain to a piezoelectric material generates an electric field. The piezoelectric effect has no direct analogue in the energy in the magnetic domain, since the magentic field is odd under time reversal and even under parity, while the electric field is even under time reversal and odd under parity.

The symmetry variables are the three rotations of the rigid director structure, $\delta \Theta_{i}$, eq. (4). Since there is a preferred direction, $\boldsymbol{n}$, which is visible in the microscope, it is appropriate to split the three rotation angles into two rotations of, and one rotation about, $\boldsymbol{n}$

$$
\delta n_{i} \equiv \epsilon_{i k j} n_{j} \delta \Theta_{k} \quad \text { and } \quad \delta \Omega \equiv n_{i} \delta \Theta_{i}
$$

where the non-commutativity relations, eq. (3), apply to a nonlinear theory with finite rotations, appropriately. Note, both variables are odd under the replacement $n_{i} \rightarrow-n_{i}$ and read in terms of the directors $\delta n_{i}=\delta_{i j} \frac{\perp}{\delta} n_{j}$ and $\delta \Omega=$ $-l_{i} \delta m_{i}+m_{i} \delta l_{i}$.

\section{Hydrodynamics of the $O_{h}$ and $O$ phases}

\subsection{Statics}

We will concentrate on the hydrodynamic effects specific to the $O_{h}$, and particularly the $O$ phase, when compared to the isotropic state.

The Gibbs relation, connecting changes of the total energy density $d \varepsilon$ with those of all hydrodynamic variables (entropy density $\sigma$, mass density $\rho$, momentum density $g_{i}$, concentration $c$, and the symmetry variables) $[39,40]$ can be written as

$$
d \varepsilon=T d \sigma+\mu d \rho+v_{i} d g_{i}+\mu_{c} d c+h_{i}^{\Theta \prime} d \Theta_{i}+\Psi_{i j}^{\Theta} d \nabla_{j} \Theta_{i}
$$

thereby defining the conjugate quantities (temperature $T$, chemical potential $\mu$, velocity $v_{i}$, relative chemical potential $\mu_{c}$, and the "molecular fields" $h_{i}^{\Theta \prime}$ and $\Psi_{i j}^{\Theta}$ ) as partial derivatives of the total energy.

Since rigid rotations of the cubic structure must not cost energy, $h_{i}^{\Theta \prime}$ is zero (in the absence of any orienting field) and only gradients of $\Theta_{i}$ (space dependent rotations 
of the cube structure) enter the total energy. In the general case the "molecular fields" can be combined into

$$
h_{i}^{\Theta}=h_{i}^{\Theta \prime}-\nabla_{j} \Psi_{i j}^{\Theta}-2 \epsilon_{i k l} \Psi_{k j}^{\Theta} \nabla_{j} \Theta_{l},
$$

where the last contribution is due to relation (3).

The form of the quadratic, Frank-type gradient energy of inhomogeneous rotations of the cube is not affected by the chirality. Therefore, the expression for ordinary cubic biaxial nematics $[36,41]$ is valid for the $O$ phase, as well. In our notation it has the form

$$
\varepsilon_{g}=\frac{1}{2} K_{i j k l}\left(\nabla_{j} \Theta_{i}\right)\left(\nabla_{l} \Theta_{k}\right)
$$

with $K_{i j k l}=K_{1} \delta_{j l} \delta_{i k}+K_{2}\left(\delta_{j k} \delta_{i l}+\delta_{l k} \delta_{i j}\right)+K_{3} Q_{i j k l}$ containing 3 independent Frank parameters.

On the other hand, linear gradient terms, absent in the $O_{h}$ phase are possible in the $O$ phase, due to the existence of $q_{0}$ (as in the cholesteric phase) and we get one contribution

$d \varepsilon_{l g}=q_{0} k_{2} \nabla_{i} \Theta_{i}=q_{0} k_{2} \epsilon_{i j k}\left(n_{i} \nabla_{j} n_{k}+m_{i} \nabla_{j} m_{k}+l_{i} \nabla_{j} l_{k}\right)$.

This expression is the analogue of the well-known linear twist term of chiral uniaxial nematics (cholesterics). There, the linear gradient term of the director results in a helical director structure as defect-free energetic minimum state. In biaxial cholesterics helical rotations of the different directors are independent and lead to frustration [7]. In the $O$ phase rotations of the directors are not independent, but have to preserve the cubic structure. Therefore eq. (14) is minimized by a single helical rotation of the cubic structure about any of the directors - which one does not matter, since all are indistinguishable. However, this rotation of the cubic structure cannot be seen directly by optical means. Only if the orientation of the cube matters for a definite experiment, the helical structure might be detected indirectly.

If there is a linear gradient term, there are also static Lehmann-type energy contributions $[42,43]$, bilinear in linear director gradients and variations of the scalar variables, $S \in\{\sigma, \rho, c\}$

$$
\begin{aligned}
d \varepsilon_{F} & =q_{0}\left(\nabla_{i} \Theta_{i}\right) \sum_{S} K^{S}(\delta S) \\
& =q_{0} \epsilon_{i j k}\left(n_{i} \nabla_{j} n_{k}+m_{i} \nabla_{j} m_{k}+l_{i} \nabla_{j} l_{k}\right) \sum_{S} K^{S}(\delta S),
\end{aligned}
$$

where the sum over $S$ comprises all scalar variables. All these terms are due to the chirality of the $O$ phase.

Static Lehmann contributions are associated in general with chiral phases for which either the building blocks (for example, the molecules) are chiral [42], or for which the chirality is due to the low symmetry of the spatial arrangement of the units [43].

The other energy contributions regarding the scalar variables in the $O_{h}$ and $O$ phase are the same as in the isotropic state and comprise the appropriate (scalar) static susceptibilities.

\subsection{Dynamics}

The dynamics is described by conservation laws for the conserved variables and balance equations for the nonconserved ones [35]. The former contain (the divergence of) the currents (e.g., the stress tensor, $\sigma_{i j}$, in the momentum conservation law, the heat and the concentration current), while in the latter case a quasi-current balances the temporal changes of the variable

$$
\frac{\partial \Theta_{i}}{\partial t}+v_{j} \nabla_{j} \Theta_{i}-\omega_{i}+J_{i}^{\Theta R}+J_{i}^{\Theta D}=0
$$

with the vorticity $\omega_{i}=\epsilon_{i j k} \nabla_{j} v_{k}$. Both, currents and quasi-currents, are additively split into a reversible (superscript $R$ ) and a dissipative part (superscript $D$ ). The irreversible part of the dynamics can be derived from the dissipation function (or the entropy production $R$, which acts as the source term in the entropy balance), while the reversible part is non-potential, requires $R=0$ (conserved entropy), and often (but not always) follows from general invariance principles [40].

The dissipative dynamics of the $O_{h}$ and $O$ phases is similar to that of the isotropic phase regarding secondrank material tensors (e.g., describing heat conduction, diffusion, and thermo-diffusion) containing one material coefficient each [36]. The viscosity tensor, relating the stress tensor $\sigma_{i j}$ with symmetrized flow, $2 A_{i j}=\nabla_{j} v_{i}+$ $\nabla_{i} v_{j}$, has the same form as $K_{i j k l}$ in eq. (13) containing three flow viscosities, one more than in the isotropic phase. There is one rotational viscosity, $\gamma_{1}$, describing relaxation of inhomogeneous cube rotations, $J_{i}^{\Theta D}=\left(1 / \gamma_{1}\right) h_{i}^{\Theta}$.

In addition, in the $O$ phase there are dissipative Lehmann-type terms due to chirality (as in cholesterics)

$$
2 R_{L}=q_{0} h_{i}^{\Theta} \sum_{P} \psi_{P} \nabla_{i} P,
$$

where the sum over $P$ comprises $\nabla_{k} P \in\left\{\nabla_{k} T, \nabla_{k} \mu_{c}, E_{k}\right\}$, the thermodynamic forces related to the thermal degree of freedom, the concentration and the electric field, with one dissipative coefficient $\psi_{P}$ for each force.

The dissipative parts of the Lehmann effect has been modeled first by Leslie [44] for classical cholesterics in the framework of a continuum-type description. Using the hydrodynamic approach [42] it became clear that such effects not only arise for cholesterics, but also for chiral smectic liquid crystals. Dissipative Lehmann effects are thus complementing the static Lehmann effects discussed in sect. 5.1 .

As a result there are contributions to the dissipative quasi-current, $J_{i}^{\Theta D}=q_{0} \sum_{P} \psi_{P} \nabla_{i} P$ indicating rotations of the cubic structure about the direction of, e.g., an applied temperature gradient, which in nematics is called Lehmann rotation $[42,43]$. In the cubic $O$ phase, however, this rotation cannot be seen directly. Variational derivatives of $R$ with respect to $\nabla_{i} P$ lead to, e.g. dissipative heat current, $j_{i}^{\sigma D}=q_{0} \psi_{T} h_{i}^{\Theta}$. In nematics, where the appropriate force $h_{i}^{n}$ can be generated by an external rotating magnetic field, this is called the inverse Lehmann effect [45]. How to excite a finite $h_{i}^{\Theta}$ and therefore a heat current will 
be discussed in the next section. In ref. [45] it has been shown that measurements of the inverse Lehmann effect and of the direct Lehmann effects discussed so far can be combined to disentangle static and dynamci contributions of the Lehmann effect.

The part of the reversible dynamics related to transport $\left(v_{j} \nabla_{i} \Theta_{i}\right)$ or convection $\left(-\omega_{i}\right)$ in eq. (16) is due to general invariance principles, like Galilean invariance and rotational covariance $[39,40]$. For biaxial nematics this has been investigated in detail in ref. [35].

In many nematic phases there is a phenomenological reversible coupling between director reorientation and symmetrized flow known as "flow alignment" and "back flow". In general, flow alignment takes in the stress tensor the form

$$
\sigma_{i j}=\lambda_{i j k} h_{k}
$$

where $\sigma_{i j}$ is the stress tensor and $h_{k}$ is the molecular field associated with variables characterizing spontaneously broken rotational symmetries, such as, for example, deviations of the director from its preferred direction. $\lambda_{i j k}$ does not lead to dissipation or the generation of heat, since $h_{k}$ is even under time reversal $[46,47]$.

In uniaxial nematics there is one phenomenological parameter (related to the flow alignment angle under shear flow). In the $O_{h}$ and $O$ phases, however, there is none due to the cubic symmetry [36].

\subsection{External fields}

Generally, external fields can orient liquid crystals. In uniaxial nematics, electric fields $E_{i}$ and magnetic fields $H_{i}$ reorient the nematic director either along the field or perpendicular to it, depending on the sign of the dielectric or magnetic anisotropy. In cubic biaxial nematics (both, in the $O_{h}$ and in the $O$ phase) there is no anisotropic quadratic term in the energy due to the high symmetry of the cubic phase. There are also no third-rank tensors. Naturally anisotropic terms of fourth order in external fields are possible for both, the $O_{h}$ and the $O$ phase; they are of the structure $\sim E_{i} E_{j} E_{k} E_{l}, \sim H_{i} H_{j} H_{k} H_{l}$ or $\sim E_{i} E_{j} H_{k} H_{l}$ and are well documented in the literature for cubic liquid crystalline systems $[41,48,49]$.

Taking, e.g., the magnetic field energy

$$
\varepsilon_{H}=-\chi_{H} Q_{i j k l} H_{i} H_{j} H_{k} H_{l}
$$

it is easy to see that the energetic minimum for $\chi_{H}>0$ is obtained, when the cube is oriented with one of its edges along the field $(\boldsymbol{l}, \boldsymbol{m}$, or $\boldsymbol{n})$, while for $\chi_{H}<0$ the field is along the space diagonal of the cube. For deviations from the equilibrium orientation, a finite energy occurs giving rise to a torque on the cube $h_{i}^{\Theta}=$ $\left(\left|\chi_{H}\right| / 3\right) \epsilon_{i q p} Q_{p j k l} H_{q} H_{j} H_{k} H_{l}$ using the inverse of eq. (2).

For the inverse Lehmann effect, a magnetic field rotating with angular velocity $\omega$ (e.g., in $x / y$ plane) drags the cube also into a rotation in this plane. There is, however, a time lag, $\Delta$, due to the rotational viscosity $\gamma_{1}$, ensuring that the orientation of the cube (for $\chi_{H}<0$ ) is never in the equilibrium position parallel to the field. For the time lag we get $\sin (4 \Delta)=-\left(12 /\left|\chi_{H}\right| H_{0}^{4}\right) \gamma_{1} \omega$, which leads to a heat current $j_{z}^{\sigma D}=q_{0} \psi_{T} h_{z}^{\Theta}=-q_{0} \psi_{T} \gamma_{1} \omega$ independent of the magnetic field strength, similar to the nematic case [43]. Although the cube rotation cannot be seen directly, the heat current due to this inverse Lehmann effect is detectable by standard means.

In a gel with $O$ or $O_{h}$ symmetry, permanent or transient elasticity is prone to electro- and magnetostriction, when external fields are present. This effect is governed by a fourth-rank tensor of the structure, eq. (13), with 3 independent material coefficients.

\section{Hydrodynamics of the $T_{h}$ phase}

The hydrodynamic variables in $T_{h}$ phases are the same as for the $O_{h}$ and $O$ phase, respectively: three independent rotation of the cubic structure, eq. (2).

In the following we will focus on the changes of the hydrodynamics in the $T_{h}$ phase when compared to the hydrodynamics of the $O_{h}$ phase.

For the static behaviour related to director deformations we find one additional Frank constant, which is associated with the contribution

$$
\varepsilon_{T_{h}}=K_{4} Q_{i j k l}^{T}\left(\nabla_{i} \Theta_{k}\right)\left(\nabla_{j} \Theta_{l}\right) .
$$

Thus instead of having three Frank constants as for $O_{h}$, eq. (13), we have now four for $T_{h}$.

There exists a third-rank tensor,

$$
\begin{aligned}
d_{i j k} \equiv & \epsilon_{i p q} Q_{q j p k}^{T}=Q_{0}\left(n_{i}\left[l_{j} m_{k}+l_{k} m_{j}\right]\right. \\
& \left.+m_{i}\left[l_{j} n_{k}+l_{k} n_{j}\right]+l_{i}\left[n_{j} m_{k}+n_{k} m_{j}\right]\right)
\end{aligned}
$$

which is symmetric in $j, k$ and is invariant under spatial inversion. By its definition it is clear that $d_{i j k}$ fulfils all symmetry requirements of the $T_{h}$ phase, since $Q_{i j k l}^{T}$ does. In the representation in terms of directors one has to take into account that $\boldsymbol{l}$ is a shorthand for $\boldsymbol{n} \times \boldsymbol{m}$ thereby leading to expressions that are manifestly symmetric under $\boldsymbol{n} \rightarrow-\boldsymbol{n}$ and $\boldsymbol{m} \rightarrow-\boldsymbol{m}$. This tensor allows to describe flexoelectricity

$$
\varepsilon_{f l}=e_{1} d_{i j k} E_{i} \nabla_{j} \Theta_{k}
$$

containing one flexoelectric coefficient. Flexoelectric contributions are, in general, static contributions coupling electric fields to gradients of the variables associated with spontaneously broken rotational symmetries. They have been introduced first for uniaxial nematics by Meyer [50] under the notation of "piezoelectricity".

All static material properties associated with secondrank tensors are the same as for $O_{h}$ and contain one parameter each due to the cubic symmetry.

For liquid crystalline gels and elastomers, where elasticity is described by the strain tensor $u_{i j}$, there are two other static material properties associated with fourthrank property tensors, which differ in the $T_{h}$ phase from the $O_{h}$ phase, namely electrostriction and magnetostriction, for which we have one additional contribution each

$$
\varepsilon_{T_{h} E M}=Q_{i j k l}^{T} u_{k l}\left(\chi^{E} E_{i} E_{j}+\chi^{H} H_{i} H_{j}\right) .
$$


This contribution comes in addition to the usual three contributions from the $O_{h}$ phase (compare ref. [22] for solid state physics). It can be investigated experimentally, for example by applying an electric field in the $x$ direction. Then the strain fields induced in the two directions perpendicular to the electric field, namely the $y$ - and $z$-directions, would be different. It is easily checked that in this case the strain difference

$$
u_{y y}-u_{z z} \sim Q_{0}^{T} \chi^{E} E_{x}^{2}
$$

arises.

Other static fourth-rank property tensors are identical in the $O_{h}$ and $T_{h}$ phase, [22], in particular those associated with the energetic contributions $\chi_{i j k l}^{E} E_{i} E_{j} E_{k} E_{l}$, $\chi_{i j k l}^{H} H_{i} H_{j} H_{k} H_{l}$, and the Hookean elasticity, $c_{i j k l} u_{i j} u_{k l}$, since the antisymmetric $Q_{i j k l}^{T}=-Q_{k l i j}^{T}$ cannot contribute. On the other hand, $\chi_{i j k l}^{E H} H_{i} H_{j} E_{k} E_{l}$ has a fourth coefficient due to $Q_{i j k l}^{T}$.

In the dynamics there is, in the $O_{h}$ and the $T_{h}$ phase, one rotational viscosity and three flow viscosities, the latter because the viscosity tensor has the same symmetry as the elastic one. In contrast to the $O_{h}$ phase, there is flow alignment and back flow in the $T_{h}$ phase

$$
\begin{aligned}
J_{i}^{\Theta R} & =\lambda^{T} d_{i j k} A_{j k}, \\
\sigma_{i j}^{R} & =-\lambda^{T} d_{k i j} h_{k}^{\Theta},
\end{aligned}
$$

indicating e.g. orientation of the cubic structure by shear flow, which is, however, difficult to detect optically.

When the $T_{h}$ phase is chiralized, a chiral $T$ phase results. It shows piezoelectricity $\varepsilon_{p} \sim q_{0} d_{i j k} E_{i} u_{j k}$ and nonlinear dielectric orientation $\varepsilon_{d} \sim q_{0} d_{i j k} E_{i} E_{j} E_{k}$, but also characteristic reversible dynamic couplings between flow and $\nabla_{k} P \in\left\{\nabla_{k} T, \nabla_{k} \mu_{c}, E_{k}\right\}$, e.g. $\sigma_{i j}^{R} \sim q_{0} d_{k i j} \nabla_{k} T$ and $j_{i}^{\sigma R} \sim q_{0} d_{i j k} A_{j k}$. The full hydrodynamics of the $T$ phase has been presented before in refs. [27,37], in a slightly different language.

\section{Hydrodynamics of the $D_{4 h}$ and $D_{4}$ phases}

We focus on the hydrodynamics of the symmetry variables $\delta n_{i}$ and $\delta \Omega$ and their couplings to other variables involving various material tensors.

Since both, the $D_{4 h}$ and the $D_{4}$ phase do not have a polar direction, there are no non-vanishing property tensors of first rank. Second-rank property tensors in both, the $D_{4 h}$ and the $D_{4}$ phase take typically the uniaxial form

$$
\xi_{i j}=\xi_{\|} n_{i} n_{j}+\xi_{\perp} \delta_{i j}^{\perp},
$$

where $n_{i}$ denotes the director associated with the preferred direction. Property tensors of this type include the dielectric tensor and the tensor of magnetic susceptibilities, in hydrostatics and in dissipative dynamics the tensors of heat conduction, electric conductivity, and diffusion.

For fourth-rank property tensors, the differences to the case of uniaxial nematics can all be traced back to the existence of the additional variable $\delta \Omega_{i}$, and of the order parameter $Q_{i j k l}^{t r}$, which is characteristic for tetragonal symmetry. For the analogue of Frank's free energy of deformations of the orientational order,

$$
\begin{aligned}
\varepsilon_{g}= & \frac{1}{2} K_{i k j l}\left(\nabla_{i} n_{k}\right)\left(\nabla_{j} n_{l}\right)+K_{7} \delta_{i k}^{t r} \epsilon_{l i j}\left(\nabla_{l} \Omega\right)\left(\nabla_{j} n_{k}\right) \\
& +\left(K_{5} n_{i} n_{j}+K_{6} \delta_{i j}^{t r}\right)\left(\nabla_{i} \Omega\right)\left(\nabla_{j} \Omega\right) .
\end{aligned}
$$

There are four Frank-type orientational elastic coefficients related to distortions of the director, i.e. one more than in uniaxial nematics, since $K_{i j k l}$ contains additionally $K_{4} Q_{i j k l}^{\perp}$, totaling the number of coefficients to seven.

Similarly, there are seven coefficients for the electroand magnetostriction tensor (in the case of gels). Another class of rank- 4 tensors are the viscosity tensor $\eta_{i j k l}$ and the elastic tensor $c_{i j k l}$ (in the case of gels), which have six coefficients. This is one more than in the uniaxial case [39, 46] and the additional coefficient, e.g. $\eta_{6} Q_{i j k l}^{\perp}$ again is due to the tetragonal transverse order. Thus, the number of coefficients in rank-4 tensors in the $D_{4 h}$ and $D_{4}$ phase is the same as in the $D_{2 d}$ phase [26,37], in accordance with [22].

There is no rank-3 tensor, which is odd under the $\boldsymbol{n} \rightarrow-\boldsymbol{n}$ transformation. Therefore, the flexoelectric energy $\varepsilon_{f l}=f_{i j k} E_{i} \nabla_{j} n_{k}$ has the same form as in the uniaxial nematic phase [5] and contributions of the form $E_{i} \nabla_{i} \Omega$ are ruled out. Similarly in the dynamics of the symmetry variables

$$
\begin{aligned}
\frac{\partial}{\partial t} n_{i}+v_{j} \nabla_{j} n_{i}+(\boldsymbol{\omega} \times \boldsymbol{n})_{i}-\lambda_{i j k} A_{j k}+X_{i} & =0, \\
\frac{\partial}{\partial t} \Omega+v_{j} \nabla_{j} \Omega-n_{i} \omega_{i}+Y^{\Omega} & =0,
\end{aligned}
$$

the flow alignment tensor, $\lambda_{i j k}$, is as in the uniaxial nematic case [46], and there is no flow alignment regarding $\Omega$.

All features discussed above apply to the $D_{4}$ phase as well, but there are additional chiral effects, which we will discuss in the following. First we inspect linear gradient terms in the generalized energy as well as static and dissipative Lehmann-type contributions. For these the arguments for the $D_{4}$ phase are quite in parallel to those for the $O$ phase, with differences originating in the different symmetries, tetragonal and cubic, involved.

For deformations of the director fields we find two linear gradient terms

$$
d \varepsilon_{l g}=q_{0}\left(\epsilon_{i j k} k_{\perp} n_{i} \nabla_{j} n_{k}+k_{\|} n_{i} \nabla_{i} \Omega\right) .
$$

Minimizing the first contribution leads to a helical rotation of $\boldsymbol{n}$ about any of the two (indistinguishable) transverse preferred directions, $\boldsymbol{m}$ or $\boldsymbol{l}$, while the second one gives a helical rotation of the transverse structure about $\boldsymbol{n}$. This latter type of helical state actually arises, if $\left|k_{\|}\right|>\left|k_{\perp}\right|$.

For the static Lehmann contributions we have, with $P \in\{\sigma, \rho, c\}$ denoting all the scalar variables,

$$
d \varepsilon_{F}=q_{0} \sum_{P} \delta P\left(K_{\perp}^{P} \epsilon_{i j k} n_{i} \nabla_{j} n_{k}+K_{\|}^{P} n_{i} \nabla_{i} \Omega\right)
$$

again with two coefficients for each variable $\delta P$. 
Table 1. We summarize the physical properties of the various phases considered here. We have also included the $T_{d}$ and $T$ phases, which show octupolar order for comparison purposes. The macroscopic physical properties listed include chirality, Lehmann effects, number of electrostrictive coefficients, piezoelectricity and flow alignment.

\begin{tabular}{|l|l|l|l|l|l|l|}
\hline Phase & Parity & Chiral & Lehmann & Electrostriction & Piezoelectric & Flow alignement \\
\hline$O(432)$ & -1 & yes & yes & 3 & no & no \\
\hline$O_{h}(m 3 m)$ & +1 & no & no & 3 & no & no \\
\hline$T_{h}(m 3)$ & +1 & no & no & 4 & no & yes \\
\hline$T_{d}(\overline{4} 3 m)$ & -1 & no & no & 3 & yes & no \\
\hline$T(23)$ & -1 & yes & yes & 4 & yes & yes \\
\hline$D_{4 h}\left(\frac{4}{m} m m\right)$ & +1 & no & no & 7 & no & yes \\
\hline$D_{4}(422)$ & -1 & yes & yes & 7 & yes & yes \\
\hline
\end{tabular}

For the corresponding dissipative Lehmann contributions in the $D_{4}$ phase we get

$$
2 R_{L}=q_{0} \sum_{S}\left(\nabla_{k} S\right)\left(\psi_{\perp}^{S} h_{k}^{n}+\psi_{\|} h_{k}^{\Omega}\right)
$$

where the thermodynamic forces $\nabla_{i} S \in\left\{\nabla_{i} T, \nabla_{i} \mu_{c}, E_{i}\right\}$ and the conjugates, $h_{i}^{n}$ and $h_{i}^{\Omega}$, follow from the appropriate part of the energy density

$$
\varepsilon=h_{i}^{n \prime} d n_{i}+\Psi_{i j}^{n} d \nabla_{j} n_{i}+h_{i}^{\Omega} d \nabla_{i} \Omega+T d \sigma+\mu_{c} d c+\ldots
$$

In the absence of external fields there is $h_{i}^{n \prime}=0$, but in the general case the full conjugate of $d n_{i}$ can be written as

$$
h_{i}^{n}=h_{i}^{n \prime}-\nabla_{j} \Psi_{i j}^{n}+2 n_{k} \Psi_{k j}^{n} \nabla_{j} n_{i}
$$

where the last contribution is due to non-commutativity of finite rotations, eq. (3). There is no external field that couples to $\delta \Omega$ and only gradients of $\Omega$ enter the energy.

Piezoelectricity has a rather specific form in the $D_{4}$ phase [22]. Taking the preferred direction $\boldsymbol{n}$ as the $z$-axis, and applying a transverse electric field in the $x$-direction, leads to a strain in the $y$ - $z$ plane, while a field in $y$ direction leads to a strain of opposite sign in the $x-z$ plane. Defining $s_{i j k}=n_{i} n_{p} \epsilon_{p j k}$ we can write the piezoelectric energy as

$$
\varepsilon_{\text {piezo }}=\frac{1}{2} q_{0} \zeta_{p}\left(s_{i j k}+s_{k j i}\right) E_{j} u_{i k}
$$

which has one piezocoefficient $\zeta_{p}$, since the strain tensor $u_{i k}$ is symmetric. The other rank-3 tensor $s_{i j k}^{\perp}=\delta_{i p}^{\perp} \epsilon_{p j k}$ does not lead to an additional contribution in the symmetrized case, eq. (36). The sign of $\zeta_{p}$ is not fixed (instead of $s_{i j k}$ one could have defined a $\tilde{s}_{i j k}$ with the opposite sign), but the two contributions for $\boldsymbol{E} \| \boldsymbol{l}$ and $\boldsymbol{E} \| \boldsymbol{m}$ always come with opposite signs.

There is a third-order orienting energy, linear in the electric field, $\boldsymbol{E}$, and quadratic in the magnetic one, $\boldsymbol{H}$,

$$
\varepsilon_{f}^{c u b}=\frac{1}{2} q_{0} \zeta_{f}^{c u b}\left(s_{i j k}+s_{k j i}\right) E_{j} H_{i} H_{k}
$$

of the same form as piezoelectricity. Taking $\boldsymbol{E} \perp \boldsymbol{H}$, the electric field orients one of the transverse directions and the magnetic fields defines a direction tilted $45^{\circ}$ to the second transverse direction and $45^{\circ}$ to $\boldsymbol{n}$. This fits to a negative dielectric anisotropy that favours $\boldsymbol{n} \perp \boldsymbol{E}$, but is always in conflict with the diamagnetic anisotropy that favours either $\boldsymbol{n} \perp \boldsymbol{H}$ or $\boldsymbol{n} \| \boldsymbol{H}$.

There are no physical effects in $D_{4 h}$ related to the parity-conserving and $n$-symmetric tensor $s_{i j k}$.

The fourth-order field energies are, for $D_{4 h}$ and $D_{4}$, of the same form as for the $O$ and $O_{h}$ phase, eq. (19), when $Q_{i j k l}$ is replaced by $Q_{i j k l}^{t r}$. They allow to orient $\boldsymbol{m}$ or $\boldsymbol{l}$, when the appropriate coefficient is positive, and $(1 / \sqrt{2})(\boldsymbol{m} \pm \boldsymbol{l})$ if it is negative.

\section{Summary}

In this paper we have analyzed cubic and non-polar tetragonal liquid crystals and gels, which do not possess octupolar order, in contrast to the systems reviewed in ref. [37]. Systems of interest therefore included $O, O_{h}$ and $T_{h}$ from the cubic class and $D_{4}$ and $D_{4 h}$ from the tetragonal class of symmetries. The physical properties of these phases including Lehmann effects, piezoelectricity, flow alignment and number of electrostrictive/magnetostrictive coefficients is summarized in table 1. For completeness as well as for comparison purposes we have also included in this table the cubic phases $T_{d}$ and $T$, which have octupolar order.

We have investigated the symmetry properties and the macroscopic behavior of cubic liquid crystals with $O$ and $O_{h}$ symmetry as they have been found recently experimentally for bicontinuous cubic liquid crystalline systems with three networks. Since the domains with one hand can reach up to $1 \mathrm{~mm}$ in size [1], our prediction that static and dissipative dynamic Lehmann type should be observable in these chiral domains in the $O$ phase. In particular, the inverse Lehmann effect can be tested experimentally: A rotating magnetic field drags along the cubic structure to also rotate. Although the latter rotation is not visible in the microscope, the temperature gradient induced by this rotation due to the inverse Lehmann effect is detectable.

For the $T_{h}$ phase, which has no longer four-fold axes of rotation, in contrast to the $O_{h}$ phase, we predict the occurrence of additional electrostrictive and magnetostrictive effects associated with the static coupling terms linear in the strain and quadratic in the external 
fields. For example, when an electric field is applied parallel to an axis of the cube, there is a strain difference induced in the two directions perpendicular to the applied field. The experimental detection of such an effect would help to identify the cubic $T_{h}$ phase, which has not yet been reported in the field of liquid crystals. As a reversible dynamic effect not present in the $O$ and $O_{h}$ phases, we find that flow alignment, reflecting the coupling between the director orientation and extensional flow involving symmetrized velocity gradients, is possible in $T_{h}$ phases. We also find that, when a $T_{h}$ phase is chiralized, a phase of $T$ symmetry, which breaks parity symmetry, in contrast to the $T_{h}$ phase, results.

We emphasize that all cubic phases discussed $\left(O, O_{h}\right.$ and $\left.T_{h}\right)$ are optically isotropic just like an isotropic liquid phase. While in some cases one can see experimentally the growth habits of a cubic phase growing in the isotropic liquid phase, our analysis provides additional possibilities to distinguish the cubic phases from the isotropic phase as well as methods to identify the three cubic phases $O$, $O_{h}$ and $T_{h}$ separately.

Comparing the macroscopic properties of the $D_{4 h}$ and the $D_{4}$ phase, it emerges that for the chiral $D_{4}$ phase static and dynamic Lehmann effects are expected to be detectable experimentally provided the domain size of domains of either hand can be made sufficiently large. It should be noted that there two types of helical structures are possible in $D_{4}$, either the preferred direction is helical, or one of the transverse direction winds about the (constant) preferred direction. The former case is detectable in the microscope, while the latter is not and such a domain looks like an achiral $D_{4 h}$ one. In crossed magnetic and electric fields the orientation of the preferred direction is always frustrated due to a cubic field-orienting energy. For liquid crystalline gels of $D_{4}$ symmetry a rather specific piezoelectric effect - the static cross-coupling between strain and electric fields, which is linear in the electric field - is predicted: taking the $z$-axis as the preferred direction, and applying a transverse electric field in the $x$-direction, leads to a strain in the $y-z$ plane, while a field in $y$-direction leads to a strain of opposite sign in the $x$ - $z$ plane.

It thus emerges that cubic and non-polar tetragonal liquid crystalline systems can be rather varied in mechanisms generating mesoscopic and macroscopic chiral domains in systems composed of achiral molecules. For chiral domains generated in optically isotropic systems made of achiral molecules only one such mechanism has been suggested so far [51]. In addition, interesting electro- and magneto-mechanical effects emerge.

Open Access funding provided by Max Planck Society. It is a pleasure to thank Carsten Tschierske and Volker Abetz for stimulating discussions and correspondence. Partial support of the work by HRB and HP through the Schwerpunktprogramm SPP 1681 "Feldgesteuerte Partikel-Matrix-Wechselwirkungen: Erzeugung, skalenübergreifende Modellierung und Anwendung magnetischer Hybridmaterialien" of the Deutsche Forschungsgemeinschaft is gratefully acknowledged.

\section{Author contribution statement}

Both authors have equally contributed to this paper.

Publisher's Note The EPJ Publishers remain neutral with regard to jurisdictional claims in published maps and institutional affiliations.

Open Access This is an open access article distributed under the terms of the Creative Commons Attribution License (http://creativecommons.org/licenses/by/4.0), which permits unrestricted use, distribution, and reproduction in any medium, provided the original work is properly cited.

\section{References}

1. C. Dressel, F. Liu, M. Prehm, X. Zeng, G. Ungar, C. Tschierske, Angew. Chem. Int. Ed. 53, 13115 (2014).

2. M. Alaasar, S. Poppe, Q. Dong, F. Liu, C. Tschierske, Chem. Commun. 52, 13869 (2016).

3. C. Tschierske, G. Ungar, ChemPhysChem 17, 9 (2016).

4. C. Tschierske, Liq. Cryst. 45, 2221 (2018).

5. P.G. de Gennes, The Physics of Liquid Crystals (Clarendon, Oxford, 1975).

6. T.C. Lubensky, Phys. Rev. A 6, 452 (1972).

7. H. Pleiner, H.R. Brand, Phys. Rev. Lett. 54, 1817 (1985).

8. G.W. Gray, J.W. Goodby, Smectic Liquid Crystals, Textures and Structures (Leonard Hill, London, 1984).

9. V. Luzzati, P.A. Spegt, Nature 215, 701 (1967).

10. V. Luzzati, A. Tardieu, T. Gulik-Krzywicki, E. Rivas, F. Reiss-Husson, Nature 220, 485 (1968).

11. P.E. Cladis, P. Pieranski, M. Joannicot, Phys. Rev. Lett. 52, 542 (1984).

12. R. Barbet-Massin, P.E. Cladis, P. Pieranski, Phys. Rev. A 31, 3912 (1985).

13. H. Stegemeyer, K. Bergmann, in Liquid Crystal of Oneand Two-Dimensional Order, edited by W. Helfrich, K. Heppke (Springer, N.Y., 1980) p. $161 \mathrm{ff}$.

14. P. Göring, S. Diele, S. Fischer, A. Wiegeleben, G. Pelzl, H. Stegemyer, W. Thyen, Liq. Cryst. 25, 467 (1998).

15. M.W. Matsen, J. Chem. Phys. 108, 785 (1998).

16. V. Abetz, T. Goldacker, Macromol. Rapid Commun. 21, $16(2000)$.

17. J. Suzuki, M. Seki, Y. Matsushita, J. Chem. Phys. 112, $4862(2000)$

18. D.R. Nelson, J. Toner, Phys. Rev. B 24, 363 (1981).

19. L.G. Fel, Phys. Rev. E 52, 702 (1995).

20. H.R. Brand, K. Kawasaki, Physica A 324, 484 (2003).

21. M. Tinkham, Group Theory and Quantum Mechanics (McGraw-Hill, New York, 1964).

22. W.P. Mason, Physical Acoustics and the Properties of Solids (D. Van Nostrand, New York, 1958).

23. L. Radzihovsky, T.C. Lubensky, Europhys. Lett. 54, 206 (2001).

24. L. Radzihovsky, T.C. Lubensky, Phys. Rev. E 66, 031704 (2002).

25. H.R. Brand, H. Pleiner, P.E. Cladis, Eur. Phys. J. E 7, 163 (2002).

26. H.R. Brand, H. Pleiner, Eur. Phys. J. E 31, 37 (2010).

27. H. Pleiner, H.R. Brand, Eur. Phys. J. E 37, 11 (2014). 
28. H.R. Brand, P.E. Cladis, H. Pleiner, Eur. Phys. J. B 6, 347 (1998)

29. H.R. Brand, P.E. Cladis, H. Pleiner, Eur. Phys. J. B 31, 147 (2003).

30. P.E. Cladis, H.R. Brand, H. Pleiner, Liq. Cryst. Today 9, 1 (1999) issue No. 3/4.

31. H. Lu, X. Zeng, G. Ungar, C. Dressel, C. Tschierske, Angew. Chem. Int. Ed. 57, 2835 (2018).

32. A.M. Levelut, C. Germain, P. Keller, L. Liebert, J. Billard, J. Phys. (Paris) 44, 623 (1983).

33. A.M. Levelut, E. Hallouin, D. Bennemann, G. Heppke, D. Loetzsch, J. Phys. II 7, 981 (1997).

34. B. Pansu, Y. Nastishin, M. Imperor-Clerc, M. Veber, H.T. Nguyen, Eur. Phys. J. E 15, 225 (2004).

35. H. Brand, H. Pleiner, Phys. Rev. A 24, 2777 (1981) sect. III

36. M. Liu, Phys. Rev. A 24, 2720 (1981).

37. H. Pleiner, H.R. Brand, Braz. J. Phys. 46, 565 (2016).

38. N.D. Mermin, T.L. Ho, Phys. Rev. Lett. 36, 594 (1976).

39. P.C. Martin, O. Parodi, P.S. Pershan, Phys. Rev. A 6, 2401 (1972).
40. H. Pleiner, H.R. Brand, Hydrodynamics and Electrohydrodynamics of Liquid Crystals, Pattern Formation in Liquid Crystals, edited by A. Buka, L. Kramer (Springer, New York, 1996) p. $15 \mathrm{ff}$.

41. P. Saidachmetov, J. Phys. (Paris) 45, 761 (1984).

42. H.R. Brand, H. Pleiner, Phys. Rev. A 37, 2736 (1988).

43. H.R. Brand, H. Pleiner, D. Svenšek, Phys. Rev. E 88, 024501 (2013).

44. F. Leslie, Proc. R. Soc. A 307, 359 (1968).

45. D. Svenšek, H. Pleiner, H.R. Brand, Phys. Rev. E 78, 021703 (2008).

46. D. Forster, T.C. Lubensky, P.C. Martin, J. Swift, P.S. Pershan, Phys. Rev. Lett. 26, 1016 (1971).

47. D. Forster, Hydrodynamic Fluctuations, Broken Symmetry, and Correlation Functions (W.A. Benjamin, Reading, Mass., 1975).

48. P. Pieranski, P.E. Cladis, T. Garel, R. Barbetmassin, J. Phys. (Paris) 47, 139 (1986).

49. H.R. Brand, Mol. Cryst. Liq. Cryst. Lett. 3, 147 (1986).

50. R.B. Meyer, Phys. Rev. Lett. 22, 918 (1969).

51. H.R. Brand, H. Pleiner, Eur. Phys. J. E 40, 34 (2017). 